\title{
Bending-Dominated Auxetic Materials for Wearable Protective Devices Against Impact
}

\author{
D. Faraci ${ }^{1}$ (1) L. Driemeier ${ }^{2} \cdot$ C. Comi ${ }^{1}$ (1)
}

Received: 16 July 2020 / Accepted: 17 November 2020 / Published online: 14 December 2020

(c) The Author(s) 2020

\begin{abstract}
Auxetic metamaterials have high energy absorption capacity and indentation resistance, due to their significant densification mechanism during compression. This study investigates the performance of structured materials in layered thin plates, with potential applications in wearable protective devices for sport activities. Two different 3D lattices, conventional and re-entrant honeycomb, are studied in detail and their dynamic behaviour is compared with that of a 2D auxetic lattice. Initially, the equivalent elastic properties of the proposed geometries are investigated at varying equivalent densities. Then a new lightweight solution of a sandwich structure with an auxetic metamaterial core is proposed for possible application to facial protective masks. Numerical impact analyses of the problem show the potential benefit of the present proposal with respect to traditional mask geometries.
\end{abstract}

Keywords Auxetic metamaterial $\cdot$ Impact absorption $\cdot$ Sandwich structure

\section{Introduction}

Metamaterials are artificial materials engineered to have specific desired properties hardly found in nature. These unusual properties are obtained from the microstructure design, rather than from chemical composition of the components.

In particular, auxetic materials are metamaterials with the unusual negative Poisson's rate property, i.e, when stretched, they expand in the direction perpendicular to the applied load, see Fig. 1a.

The auxetic materials are an emerging class of materials, of great interest in many fields $[3,8,14,19]$, due to special enhanced characteristics. Auxeticity is naturally found in some biomaterials, such as healthy tendons [11] or in a cancellous bone in the proximal epiphysis of the human tibia [24] or in alpha-cristobalite structure of silicon dioxide [26].

D. Faraci

david.faraci@polimi.it

C. Comi

claudia.comi@polimi.it

1 Department of Civil and Environmental Engineering, Politecnico di Milano, Milan, Italy

2 Department of Mechatronics, University of São Paulo, São Paulo, Brazil
One of the most common auxetic structures, the so called re-entrant honeycomb geometry illustrated in Fig. 1b, was first proposed in [20]. The design of the 3D re-entrant honeycomb auxetic cellular structure is presented in [21, 23, 25]. As shown in [15] and [1] by indentation tests on copper foams and on microporous polyethylene, there is an enhancement in hardness when the Poisson's coefficient becomes negative. Several recent numerical and experimental studies show the effectiveness of auxetic lattices for impact absorption $[6,12,17,22]$. The optimal geometry of the unitary cell of an auxetic material depends on the application and it is largely studied in the literature [16, 25, 27]. In particular, it is possible to tune the equivalent Poisson's ratio, hence obtaining different mechanical properties, through the variation of the unit cell geometric parameters, as in [10,13].

Although auxetic materials are known and studied since decades, the challenge in manufacturing process of the rigorously designed 3D microstructure of metamaterials slowed the technological progress in the past. Nowadays, the development of additive manufacturing (AM) technologies, and in particular $3 D$ printing, paves the way to easy and costeffective production of metamaterials [22, 25].

The high performance of $3 \mathrm{D}$ auxetic materials in terms of drapeability and impact absorption together with the high stiffness-to-density ratio of all bending-dominated 


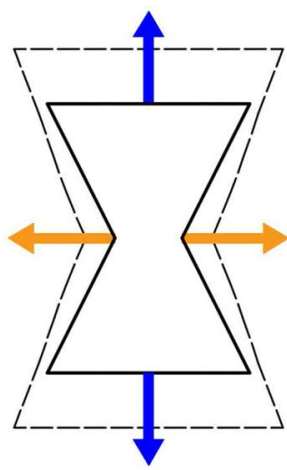

(a)
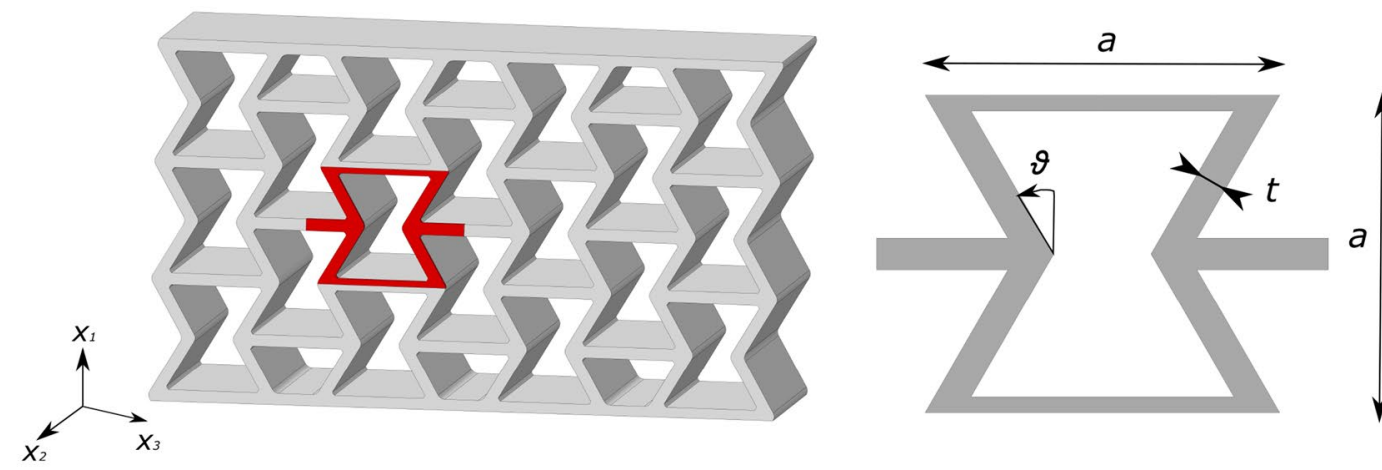

(b)

Fig. 1 a Undeformed (continuous) and deformed (dashed) shapes showing the 2D auxetic behaviour in traction (blue arrows); b 2D re-entrant honeycomb auxetic structure and unit cell with dimensions

micro-structured materials make them good candidates for sport wearable protector devices, such as facial masks.

Facial protectors attenuate and distribute the impact energy over the face. The geometry of the protector and anchoring points on the face should ensure comfort in order not to compromise the athlete's performance during practice and competition. Moreover, nasal protectors should reinforce resistance zones in the face and minimize the amount of energy that reaches the fragile nasal portion of zygomatic bones. The quality of the protection strongly depends on the mechanical properties of the material, which should have an adequate shock-absorbing capacity.

As a first step towards the optimal design of facial protector devices made of structured metamaterials, in the present work, we propose a sandwich structure with a core made of structured materials and we study its dynamic response to the impact of a rigid sphere.

Different auxetic lattices are considered. First the experimental results reported in [22] are simulated and a comparison between the original 2D lattice and the new 3D lattice is performed. Then we consider the new sandwich structure with the 3D auxetic metamaterials core. Two different metamaterials with auxetic re-entrant honeycomb structure and with honeycomb structure are analysed. In the following section, the geometry of the proposed lattices is detailed and the equivalent elastic properties are computed. Then, the impact problems to be analysed are defined. Finally, the results of the validation test and those of the impact of a rigid sphere on the newly proposed sandwich structure are presented. A comparison is made between the traditional solutions and the innovative proposal of a sandwich structure with a metamaterial core.

\section{Re-entrant Honeycomb and Honeycomb 3D-Structured Materials}

Two different 3D periodic lattice materials are considered: the re-entrant honeycomb (RHC) and the honeycomb (HC). Their regular patterns in the microstructure are defined by the unit cells shown in Figs. 2 and 3, respectively. Accordingly, the RHC may exhibit an equivalent global auxetic behaviour, while for $\mathrm{HC}$ structure, a mixed, auxetic and traditional (nonauxetic) behaviour is expected.

In both cases the unit cell is a cube of side $a$, the beams inside the cell have square cross section of side $t$ and are vertical ( $x_{1}$ direction) or inclined of $\vartheta$ with respect to the horizontal plane $\left(x_{2}-x_{3}\right)$. To avoid interpenetration of the beams, $\vartheta$ must vary between 0 and $\pi / 4$ and the ratio $\xi=t / a$ has to fulfil:

$\xi \leq \frac{\cos \vartheta-\sin \vartheta}{2(1-\sin \vartheta)}$

The equivalent mass density $\rho_{e q}$, defined as the mass of the unit cell divided by its volume, depends on $\vartheta$ and on $\xi$ according to the following equation:

$\frac{\rho_{e q}}{\rho}=\frac{2 \xi^{2}}{\cos \vartheta}[4+\cos \vartheta \pm \sin \vartheta-2 \xi(3 \pm \sin \vartheta)]$

where $\rho$ is the mass density of the bulk material and \pm has to be interpreted as + for the RHC lattice, as - for the HC one. In view of the limitations on $\vartheta$ and $\xi(1)$, the bulk material condition $\rho_{e q}=\rho$ cannot be reached. Figure 4 shows the variation of the effective mass as a function of the angle $\vartheta$ and of the ratio $\xi$ for the two lattices in the geometrically admissible region (left and middle plots). The white lines 

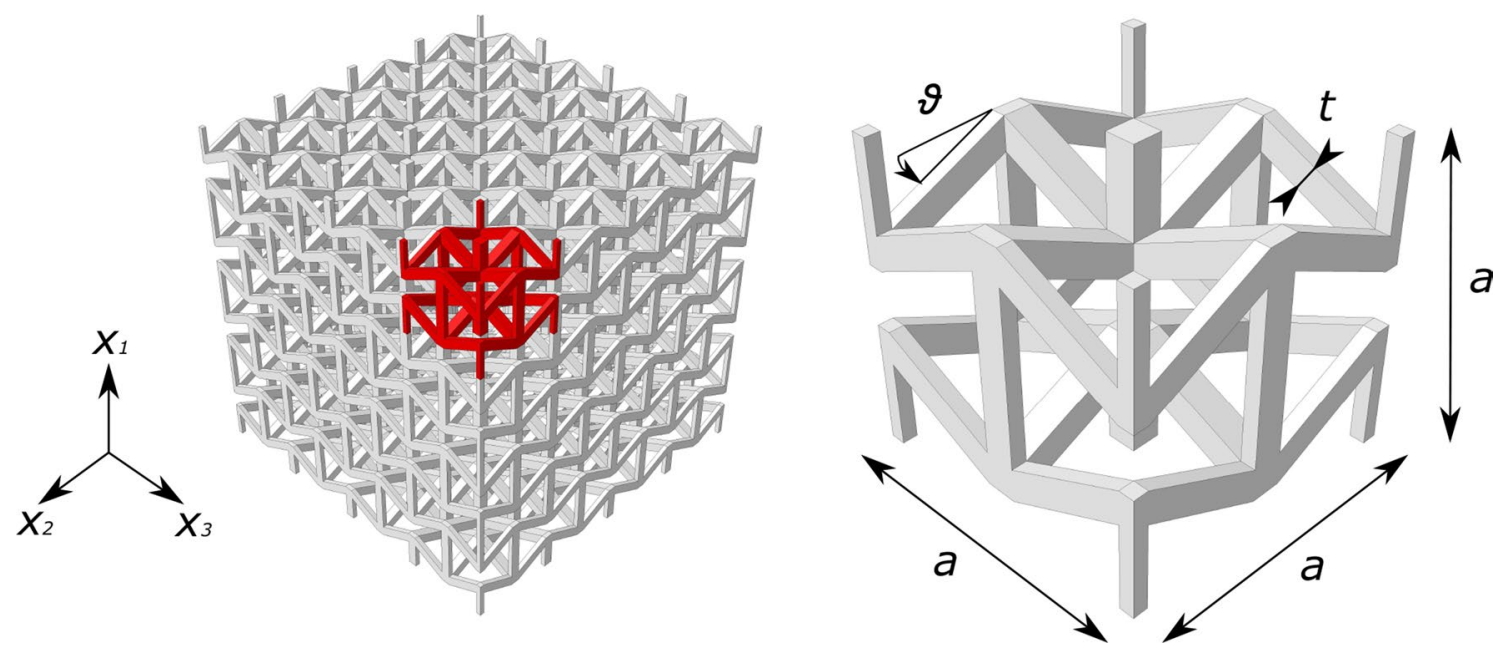

Fig. 2 RHC-structured material (left) and unit cell (right) with $\vartheta=25^{\circ}$ and $\rho_{e q}=0.1 \rho$
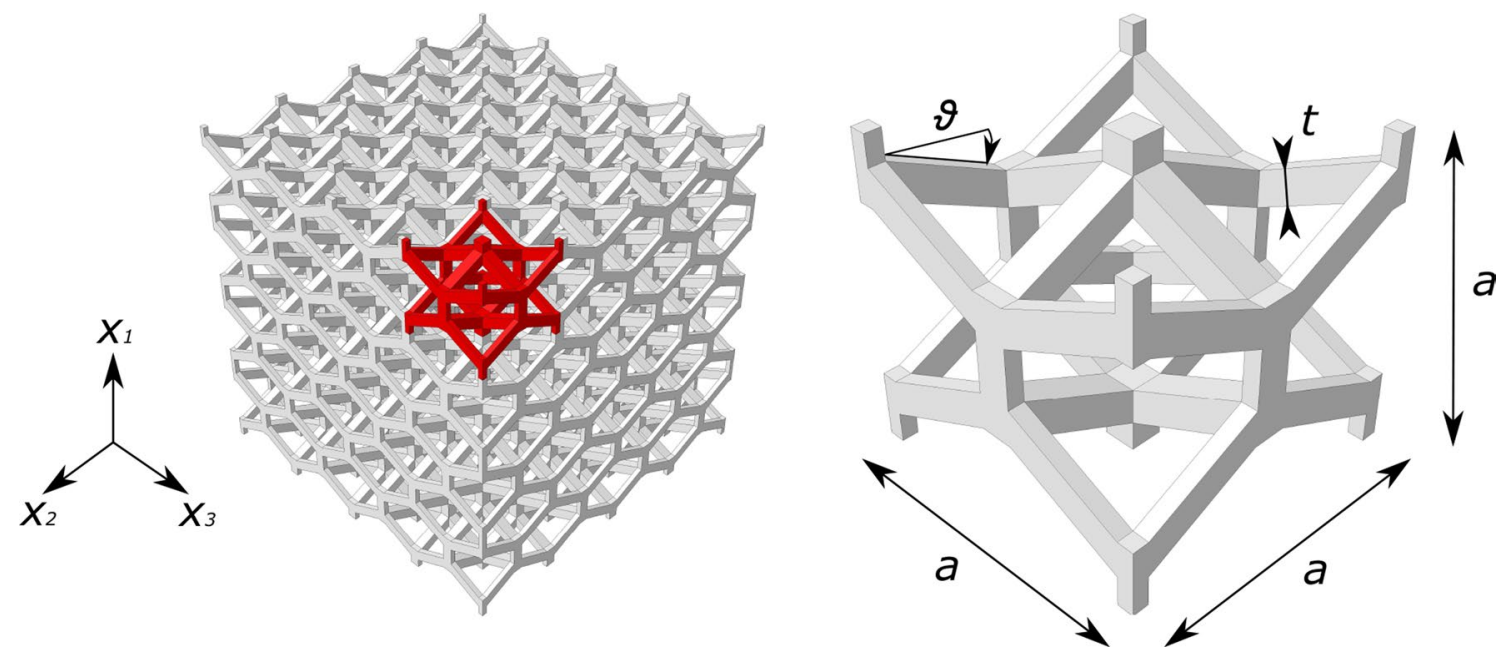

Fig. 3 HC-structured material (left) and unit cell (right) with $\vartheta=25^{\circ}$ and $\rho_{e q}=0.1 \rho$
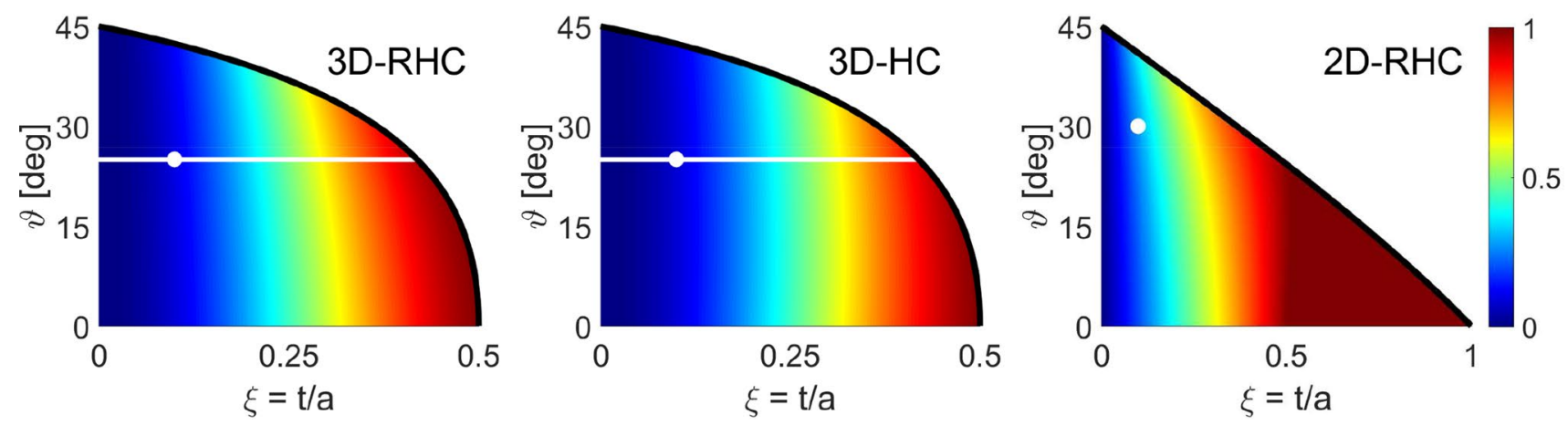

Fig. 4 Contour of the normalized equivalent mass density $\rho_{e q} / \rho$, for the three structured materials considered, as a function of angle $\vartheta$ and ratio $\xi=t / a$ 
correspond to the angle $\vartheta=25^{\circ}$ that will be considered in following, and white dots mark the equivalent mass density $0.1 \rho$ which will be used in the sandwich structure of the protective device.

For the sake of comparison, we also consider the auxetic lattice with two-dimensional structure analysed in [22] and shown in Fig. 1b. With this 2D lattice, the equivalent mass density for $0<\vartheta<\pi / 4$ reads

$\frac{\rho_{e q}}{\rho}=\xi \frac{2 \cos \vartheta+2-5 \xi \sin \vartheta}{2 \cos \vartheta-\sin \vartheta}$.

The contour plot of the equivalent density (3) is shown in Fig. 4 (rigth) in the admissible region $\xi \leq \cos \vartheta-\sin \vartheta$. The white dot marks the geometry used in [22] with equivalent mass density $0.28 \rho$ which will be used in the validation example.

The stiffness properties of the metamaterial depend on the microstructure besides on the stiffness of the constituent material. For a fixed geometry of the unit cell, the equivalent elastic properties of the 3D RHC and HC structures can be computed by means of numerical homogenization, as done e.g. in [9]. In this work, the analyses are carried out by finite elements on the unit cells of the structured materials with prescribed periodic boundary conditions in order to reproduce the behaviour of the infinite periodic lattices.

Different geometries, with inclination $\vartheta=25^{\circ}$, endowed with different equivalent densities (i.e. with a different ratio t/a) are considered. In view of the following application, we consider RHC- and HC-structured materials made of a rigid ethylene vinyl acetate (rigid EVA). The mechanical

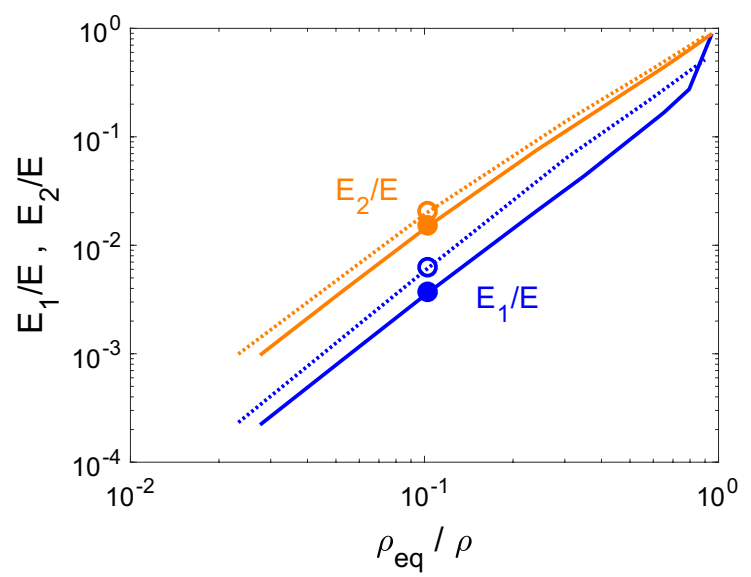

(a)

Fig. 5 a Normalized equivalent Young's modulus $E_{1} / E$ (blue) and $E_{2} / E$ (orange) and $\mathbf{b}$ equivalent Poisson's ratios $v_{21}$ (blue) and $v_{23}$ (orange), versus equivalent density $\rho_{e q} / \rho$ for the elementary RHC cell behaviour of this material has been experimentally characterized in [5]. For low levels of stress, the behaviour can be described by a linear elastic isotropic model characterized by Young's modulus $E=480 \mathrm{MPa}$ and Poisson's ratio $v=0.48$, as done in [7].

Given the selected geometries, made of an isotropic bulk material, the behaviour of the two lattices is transversely isotropic, with $x_{2}-x_{3}$ being the plane of isotropy. In the small strain hypothesis, the problem is fully linear.

The homogenized linear elastic properties for the different analysed geometries are graphically represented in Fig. 5 as function of the non-dimensional equivalent mass density $\rho_{e q} / \rho$.

Figure 5a shows the equivalent Young's modulus in the direction $x_{1}$ and $x_{2}, E_{1}$ and $E_{2}$ respectively, normalized with the Young's modulus of the bulk material $E$, for the two lattices of Figs. 2 and 3. In the log-log plot, the values are aligned on straight lines of slope $\simeq 2$; hence the equivalent stiffness is proportional to the square of the effective mass. This result is in agreement with what is reported in Ashby's charts [2] for bending-dominated metamaterials such as the ones considered in this work.

The equivalent Poisson's ratios of the two lattices are shown in Fig. 5b. The Poisson's ratio $v_{21}$ is always positive for the HC material (blue dotted line), while for the RHC (blue continuous line), it is negative for low values of effective mass density and it becomes positive for high values of the equivalent density. In the plane of isotropy, the Poisson's ratio $v_{23}$ (orange lines) is similar for the two lattices, negative for low values of the equivalent density and positive for high values. The homogenized properties tend to the elastic

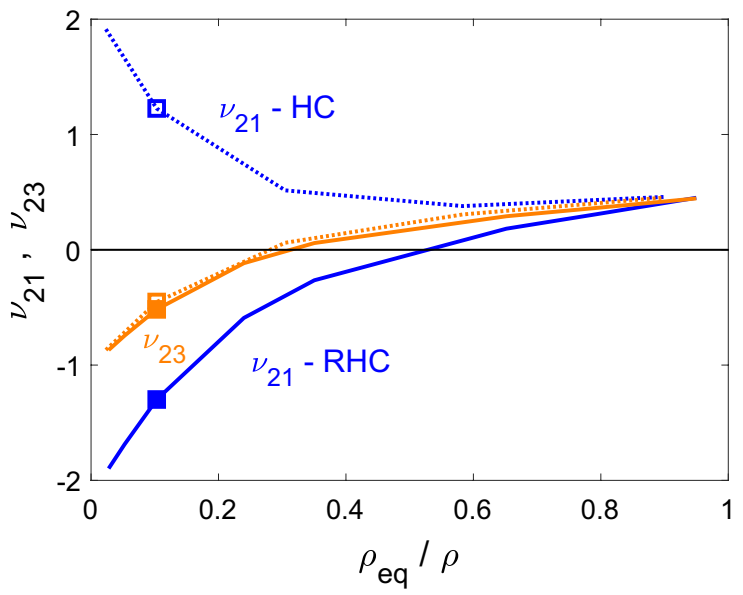

(b)

(continuous lines) and the $\mathrm{HC}$ one (dotted lines) with $\vartheta=25^{\circ}$. Markers correspond to cells with $\rho_{e q}=0.1 \rho$ 
properties of the bulk material as the equivalent mass density increases.

The equivalent bulk modulus can be computed using the relation between the elastic constants of a transversely isotropic material:

$\frac{1}{K_{e q}}=\frac{1-2 v_{12}}{E_{1}}+\frac{2\left(1-v_{21}-v_{23}\right)}{E_{2}}$

Negative values of the Poisson's ratios result in low values of the equivalent bulk modulus; therefore, the structured RHC material is more compressible than the constituent material and also than the HC-structured material. In Fig. 6, the equivalent bulk modulus of the two lattices is plotted in bilogarithmic scale. For low values of the equivalent density, the bulk modulus of the RHC material (continuous line) is two orders of magnitude lower than the one of the HC material (dotted line).

Two structured materials with equivalent mass density $0.1 \rho$ and equivalent elastic properties as marked in Figs. 5 and 6 are selected for applications in protective equipment devices. The RHC structure exhibits a full auxetic behaviour, while the $\mathrm{HC}$ one exhibits a mixed behaviour.

The metamaterial geometry is chosen as a good compromise between the practical conflicting requirements of low weight and sufficiently high mechanical stiffness of the structure.

For validation purposes, we also consider the lattice in Fig. 1b. With a cell of $21.3 \mathrm{~mm} \times 15 \mathrm{~mm}$ and wall thickness of $1.5 \mathrm{~mm}$, the equivalent mass density of the metamaterial is $\rho_{e q}=0.28 \rho$; considering a bulk material with $v=0.48$, the equivalent Poisson's ratio in the plane $x_{1}-x_{3}$ is $v_{13}=-0.338$.

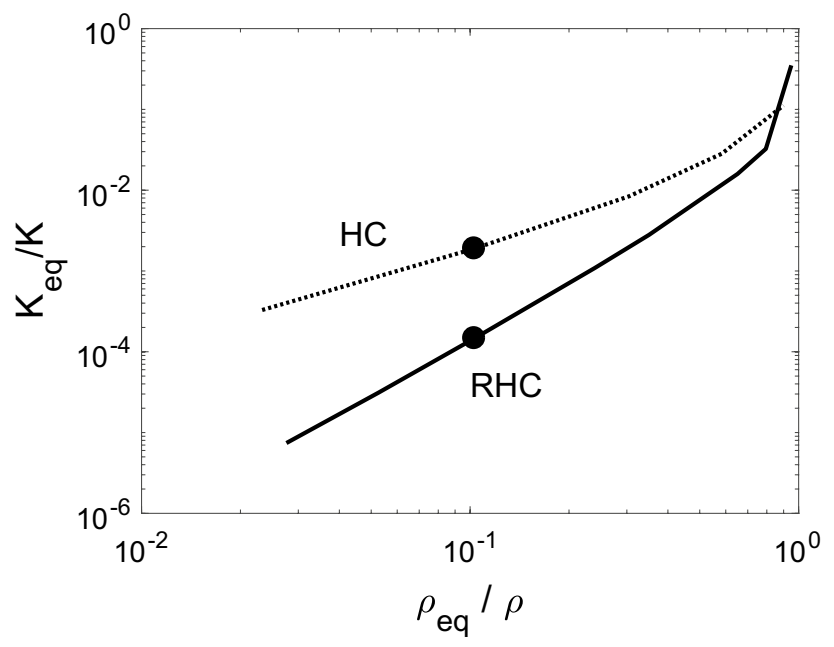

Fig. 6 Normalized equivalent bulk modulus versus normalized equivalent density $\rho_{e q} / \rho$ for the elementary RHC cell (continuous line) and the $\mathrm{HC}$ one (dotted line)
It is noted that the above-described equivalent homogenized transversely isotropic materials can be used only in the small strain regime. When geometric nonlinearity is considered, the real response of the metamaterial significantly differs from that of the equivalent homogeneous material. In particular, when compressed, instabilities of the elements and self-contact within the cell occur, the material densifies and the nonlinear equivalent stiffness increases. This property is of particular relevance for the shock-absorbing performance of this structured material.

\section{Impact on Auxetic Metamaterials}

\section{Validation Example}

We first consider one of the experimental tests reported in [22], namely, the impact of an hemisphere on the auxetic material shown in Fig. 1b. The overall dimensions of the metamaterial are $80.6 \mathrm{~mm} \times 46.5 \mathrm{~mm} \times 40 \mathrm{~mm}$ and the wall thickness is $1.5 \mathrm{~mm}$. The lattice is made of a thermoplastic polyurethane that can be modelled as a nonlinear viscous hyperelastic material. The tests in [22] are performed by a drop hammer with hemispherical head attached to a carriage that impacted the lattice with different energies.

Here, we simulate the most severe impact test with the finite element software ABAQUS explicit, considering the contact between the hammer and the lattice and the selfcontact between the walls of the lattice. The material is modelled as in [22] by the Mooney-Rivlin 5-parameter model with a viscous contribution in the form of Prony series.

We also simulate the impact on the auxetic three-dimensional material of Fig. 2 studied in this work. The cell dimension $a=14.5 \mathrm{~mm}$ and element thickness $t=1.5 \mathrm{~mm}$ is selected, leading to $\rho_{e q}=0.109 \rho$.

\section{Sandwich Structure for Wearable Protective Devices}

The problem considered here is related to the design of protector masks to be used by athletes after facial injury or to prevent it. An important aspect to be considered is the choice of material used for the mask, which must have adequate shock absorption capacity and guarantee the necessary comfort to the user. The functional requirement for the real application sets a limit on the thickness of the device (maximum value equal to $4.5 \mathrm{~mm}$ ), while the ultimate stress on the zygomatic bone sets a limit to the transmitted force in case of impact [18]. In this context, [7] proposes a mask made of two layers of flexible and rigid ethylene vinyl acetate (EVA) and compares the stresses on the skin and on the bone in case of impact with and without the mask. In [5] several solutions for nose protector made of different layers of EVA are proposed and compared. 
In the following, a different solution is proposed with the inclusion of an intermediate layer of structured material. The sandwich thin structure has three layers: two layers of homogeneous material and a central core composed by a structured material, with RHC or HC lattice. The external layer and the core are composed of rigid EVA, while the internal layer, in contact with the face, is made up of flexible EVA. As a first step towards the real design of the mask, the impact of a rigid sphere on a small portion of the structure is considered. Figure 7 illustrates the geometry and the materials of the proposed structure, for the case of RHC lattice with $a=1 \mathrm{~mm}$ and $\rho_{e q}=0.1 \rho$.

The elastic behaviour of rigid EVA can be assumed to be linear and isotropic until the yield limit $\sigma_{y}=46 \mathrm{MPa}$ is reached. Then a plastic behaviour with a very low hardening is assumed. The flexible EVA has a nonlinear elastic behaviour that, according to [5], can be described by the Ogden's model with elastic energy:

$\omega=\sum_{i=1}^{2} \frac{2 \mu_{i}}{\alpha_{i}^{2}}\left(\bar{\lambda}_{1}^{\alpha_{i}}+\bar{\lambda}_{2}^{\alpha_{i}}+\bar{\lambda}_{3}^{\alpha_{i}}-3\right)+\sum_{i=1}^{2} \frac{1}{D_{i}}\left(J_{e l}-1\right)^{2 i}$

where $\bar{\lambda}_{1}, \bar{\lambda}_{2}$ and $\bar{\lambda}_{3}$ are the deviatoric principal stretches, $\mu_{1}$, $\mu_{2}, \alpha_{1}$ and $\alpha_{2}$ are material moduli and coefficients defining the deviatoric elastic energy, $D_{1}, D_{2}$ are parameters defining the volumetric elastic energy (in the simulation nearly incompressible volumetric behaviour is assumed) and $J_{e l}$ is the elastic volumetric strain. The material properties of rigid and flexible EVAs are listed in Table 1.

In order to evaluate the performance of the proposed geometry in terms of energy absorption, we simulate the impact of a rigid sphere of $3 \mathrm{~mm}$ radius, $10 \mathrm{~g}$ mass and initial velocity of $1.5 \mathrm{~m} / \mathrm{s}$, against the sandwich structure of dimensions $8 \mathrm{~mm} \times 8 \mathrm{~mm} \times 4.5 \mathrm{~mm}$.

The following analyses allow us to compare the dynamic performance of the proposed structure with the more usual

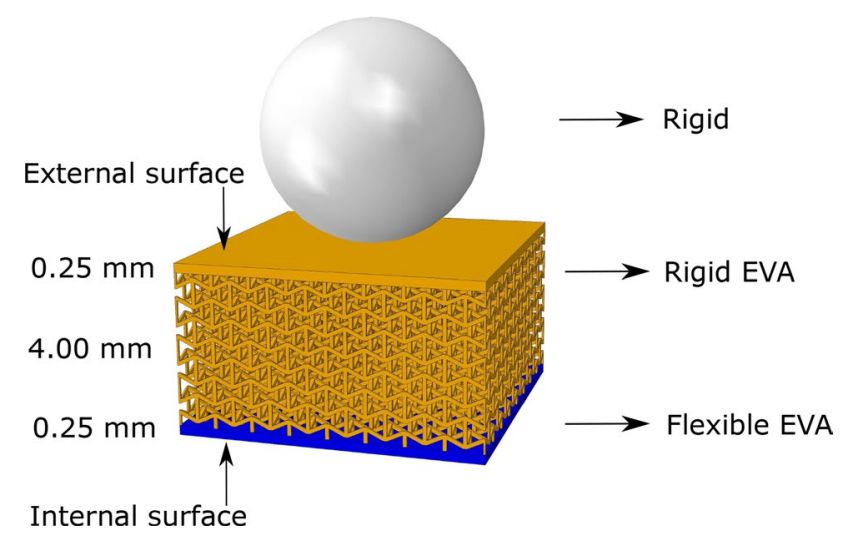

Fig. 7 Geometry of the portion of the sandwich structure, with RHC core, analysed under the impact of a rigid ball
Table 1 Mechanical properties of the constituent materials

\begin{tabular}{lll}
\hline Material & Mass density & Properties \\
\hline Rigid EVA & $\rho=940 \mathrm{~kg} / \mathrm{m}^{3}$ & $E=480 \mathrm{MPa}$ \\
& & $\nu=0.48$ \\
Flexible EVA & $\rho=2000 \mathrm{~kg} / \mathrm{m}^{3}$ & $\mu_{1}=7 \mathrm{MPa}$ \\
& $\mu_{2}=2.6 \mathrm{MPa}$ \\
& $\alpha_{1}=0.8$ \\
& $\alpha_{2}=2.6$ \\
\hline
\end{tabular}

one constituted by stratified layers of homogeneous material. In particular, the layer configurations studied in [5] and detailed in Table 2 are simulated.

The 3-dimensional dynamic simulation is conducted with ABAQUS explicit using solid elements. The metamaterial is meshed by second-order 10-node tetrahedra, while 8-node bricks, with reduced integration and hourglass control, are used for the homogeneous layers. The global mesh, with 380,000 nodes, is very refined in the contact region. An isotropic friction coefficient of 0.2 is considered to model the contact between the sphere and the structure and among the beams of the microstructure. Actually, this self-contact is essential for capturing densification, an important characteristic of the auxetic material in compression.

\section{Results}

\section{Validation Test}

The comparison between the numerical analyses here performed and the results in [22] are reported in Fig. 8a in terms of evolution in time of the reaction force. Fairly good agreement is found between our simulation with ABAQUS explicit, shown by the dotted blue line, and the experiments or analysis with LS-DYNA of [22] (dashed and continuous orange lines, respectively).

In the same figure, the response to the impact of the 3D RHC lattice here proposed, made of the same material, is

Table 2 Layers of the three stratified masks considered, from the external to the internal surface

\begin{tabular}{lll}
\hline Configuration & Material & Thickness $(\mathrm{mm})$ \\
\hline G1 & Flexible EVA & 1 \\
& Rigid EVA & 1 \\
& Flexible EVA & 2 \\
G2 & Rigid EVA & 1 \\
& Flexible EVA & 3 \\
G3 & Rigid EVA & 1 \\
& Flexible EVA & 2 \\
\hline
\end{tabular}




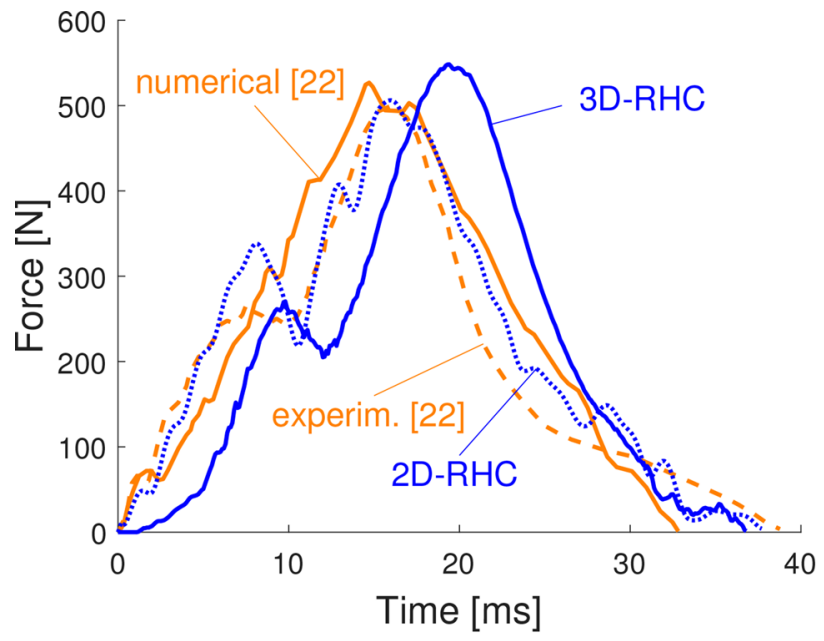

(a)

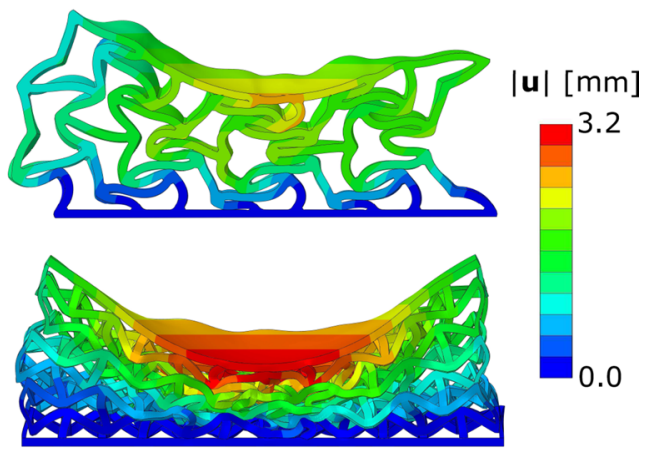

(b)
Fig. 8 Impact on metamaterials at 5J. a Evolution in time of reaction force: experimental results and numerical simulation from [22] (orange dashed and continuous lines) and present simulation with the

also shown by the continuous blue line. The 3D lattice, with global dimensions similar to the 2D lattice, is endowed with a significantly lower mass density. In particular, in the example considered here, the weight reduction is of $46 \%$, while the peak force is only slightly increased (8\%), attesting the effectiveness of the proposed 3D geometry for lightweight impact protector devices. After the impact with the 2D lattice, the kinetic energy of the hemisphere is reduced to 0.75 of the initial value, while with the $3 \mathrm{D}$ lattice a higher reduction, to 0.63 of the initial value, is obtained.

Figure $8 \mathrm{~b}$ shows the deformed configurations of the two lattices at the instant of peak force: the instability of the beams and the self-contact inside the two lattices is evident. These instabilities cause the oscillation in the force evolution: the actual location and sequence of the instabilities depend on the details of the mesh and of the contact algorithm and hence in our simulation differ from the original one; however, the global numerical response is similar, as shown in Fig. 8a.

\section{Portion of the Protective Device}

Figure 9 shows the contour plot of the displacement magnitude at different instants during the impact for both lattices on the deformed configuration (left plots: RHC, right plots: HC) of the core of the sandwich structure. One can observe that very large displacements are induced in the structure, which activate contact between the different portions of the lattices below the impact point. Furthermore, the deformed configuration is non-symmetric and hence a full $3 \mathrm{D}$ analysis is required. same 2D RHC lattice (blue dotted) and with the new 3D RHC lattice (blue continuous); $\mathbf{b}$ deformed configuration at the peak of the force of the 2D (top) and 3D (bottom) lattices

The results of the impact analyses considering RHC and $\mathrm{HC}$ lattices are compared in Fig. 10a in terms of total reaction force transmitted through the internal surface of the device. The auxetic configuration, through a densification mechanism, turns out to be more effective to protect from the impact. In fact, the maximum force transmitted is lower when adopting a protective structure with RHC lattice (continuous line): $17 \mathrm{~N}$ instead of the $27 \mathrm{~N}$ obtained with the HC lattice (dotted line).

Figure 10b shows the time evolution of energy contributions; continuous and dotted lines refer to RHC and HC lattices, respectively. The kinetic energy (blue curves) is zero at the instant that the ball rebound initiates, $1.0 \mathrm{~ms}$ for the $\mathrm{HC}$ and $1.35 \mathrm{~ms}$ for RHC, when the force transmitted is maximum. The total elastic strain energy (orange curves), integrated in time, is higher for the RHC lattice as significant deformations occur for a longer time frame. The plastic dissipation contribution (black curves) is limited in both cases.

Figure 11a shows the time histories of the transmitted force for the three configurations with the bulk-stratified materials presented in Table 2. The best configuration is G1, with a maximum force of $77 \mathrm{~N}$. The advantage of using a structured material is clearly evidenced in Fig. $11 \mathrm{~b}$ where the response of the innovative solutions, showed by black lines, is compared with those obtained with bulk materials, orange lines. With the RHC lattice, a reduction in the peak force of $78 \%$ with respect to the G1 configuration is obtained. The structured material also reduces the weight of the mask: the configuration showed in Fig. 7, with a RHC lattice core, weights about a sixth of the G1 configuration. 

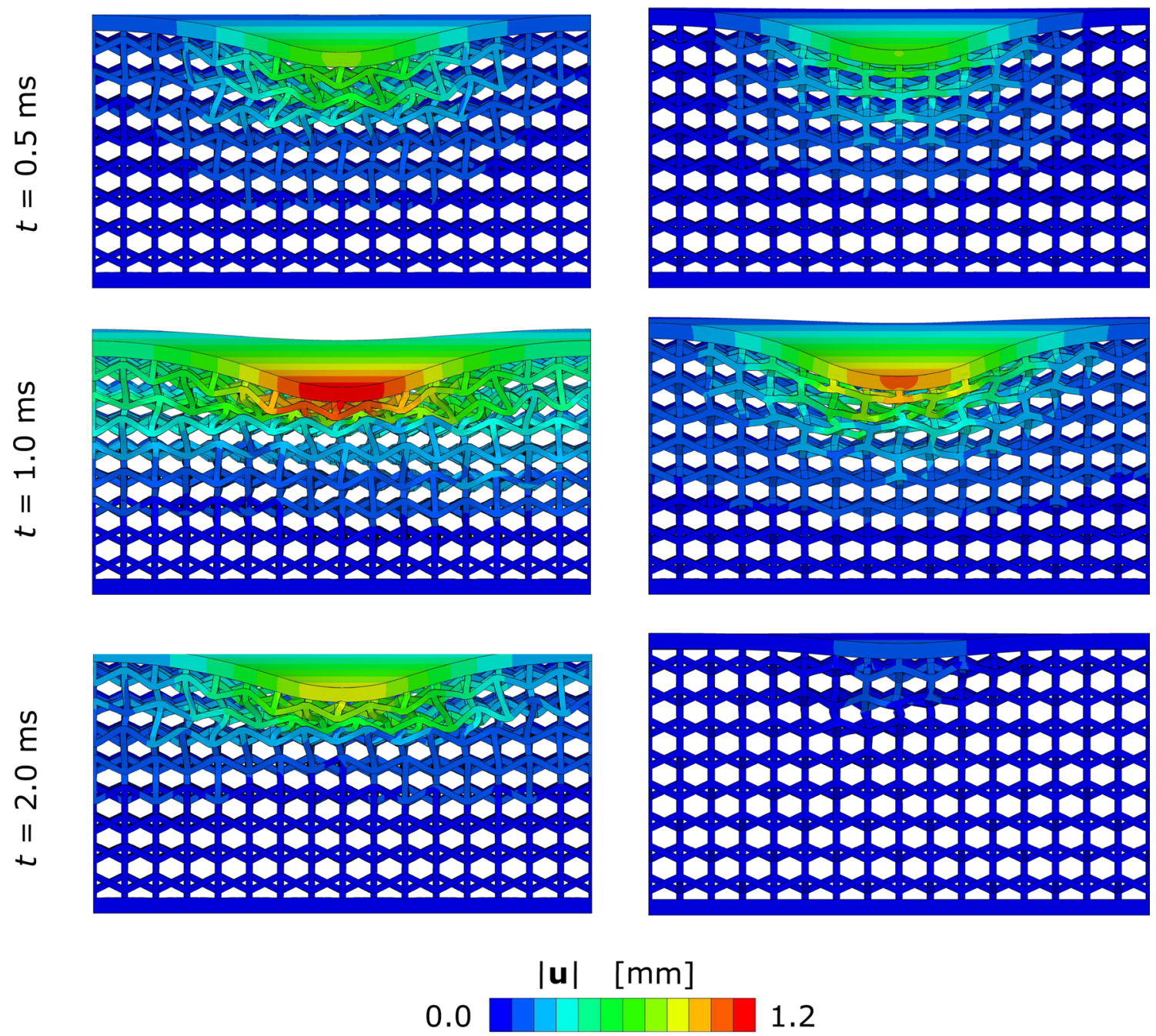

Fig. 9 Displacement magnitude at three instants during impact; left column: RHC lattice, right column: HC lattice

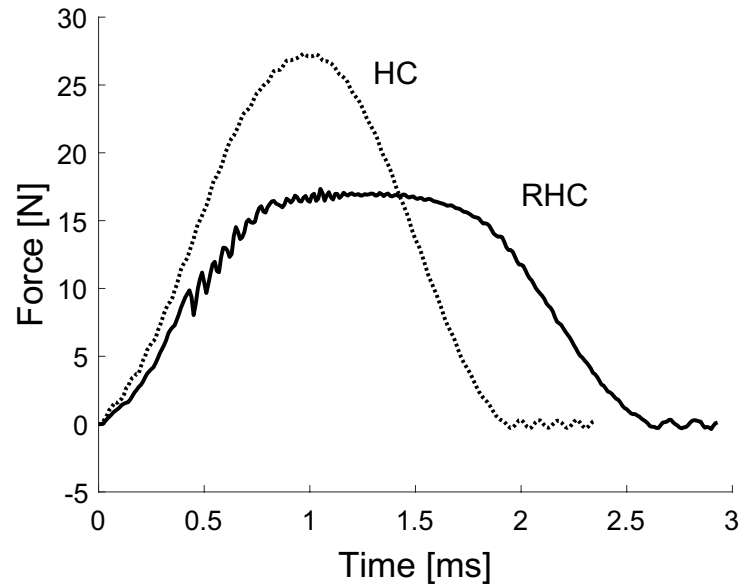

(a)

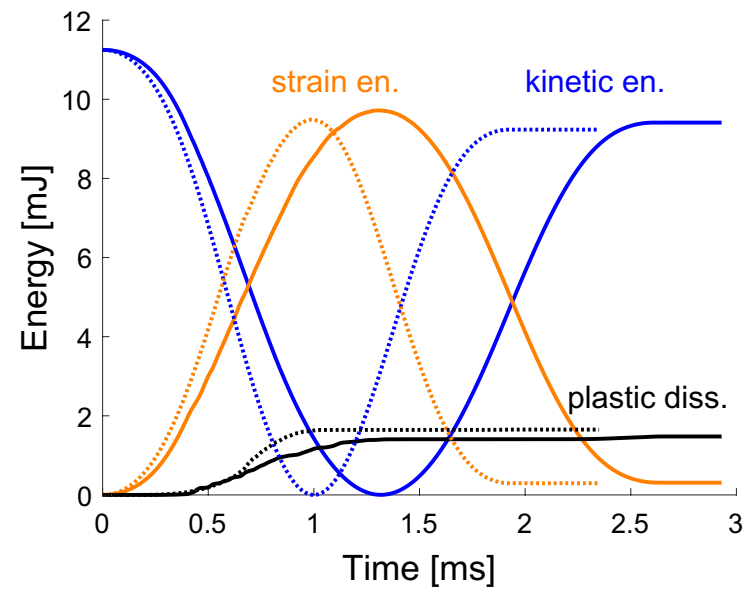

(b)

Fig. 10 a Time evolution of the reaction force at the internal surface of protection devices; $\mathbf{b}$ time evolution of kinetic energy (blue), elastic energy (orange) and plastic dissipation (black). Solid lines: RHC lattice, dotted lines: HC lattice 


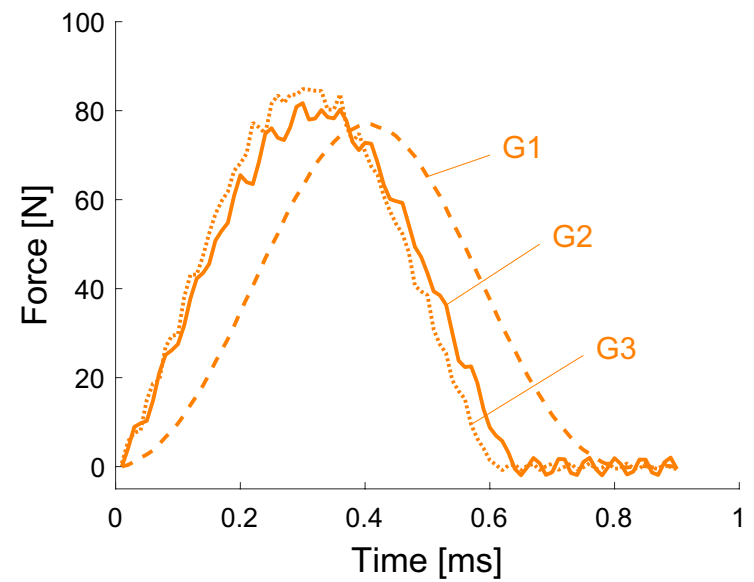

(a)

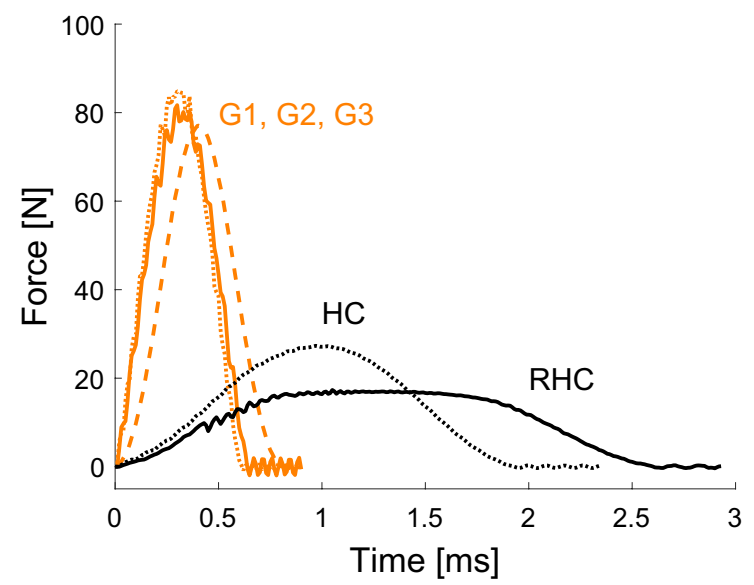

(b)

Fig. 11 a Time history of transmitted force for different protection devices with bulk materials: G1 (dashed line), G2 (continuous line) and G3 (dotted line). b Comparison between transmitted forces by

As a further confirmation of the beneficial effect of the structured material, in Fig. 12, the maximum compression stress at the internal surface of the device is shown for the different configurations. In the case of a facial protective mask, these compression stresses are transmitted to the face and their values should be minimized to protect the athlete. Again, the configuration proposed here with the RHC material has the best performance, with maximum compression of $-1.8 \mathrm{MPa}$; for the G1 configuration, the maximum compression is $-2.8 \mathrm{MPa}$. Despite a higher value of the maximum force, the $\mathrm{G} 2$ configuration allows for a reduction of the maximum compression stress with respect to the G1 configuration, with a value of $-2.6 \mathrm{MPa}$, as already remarked in [5].

\section{Conclusions}

In this paper, we propose the use of auxetic metamaterials to design lightweight stratified structures with efficient impactabsorbing properties. To this purpose, two different sets of $3 \mathrm{D}$ bending-dominated lattices are investigated. Numerical analyses conducted on a single cell of different lattices, at varying effective mass densities, allow to characterize the

stratified structure (orange curves) and structure with RHC (black continuous line) and HC (black dotted line) metamaterials

equivalent transversal isotropic behaviour and to evidence the auxetic effect that arises at low equivalent density.

We select two lattices, characterized by a low value of the effective mass density and by a strong complete or partial auxetic behaviour, to constitute the core of the sandwich structure for new protection devices. The impact analyses on a portion of this structure show a significant reduction of the force level and of the stress transmitted, indicating the potential advantages of the proposed solution.

The results presented here can open the way to the design of new light and efficient wearable protective devices such as facial protector masks. The actual fabrication of these complex geometries, however, is challenging. It requires proper advanced additive manufacturing techniques at small scale and it is currently under study. Besides 3D printing, a promising technique for very small dimensions (in the range of tens of microns) is represented by the two-photon polymerization technique [4].

The simulation of the real whole mask composed by the structured material would require a proper multiscale approach and further research is required in this direction. 


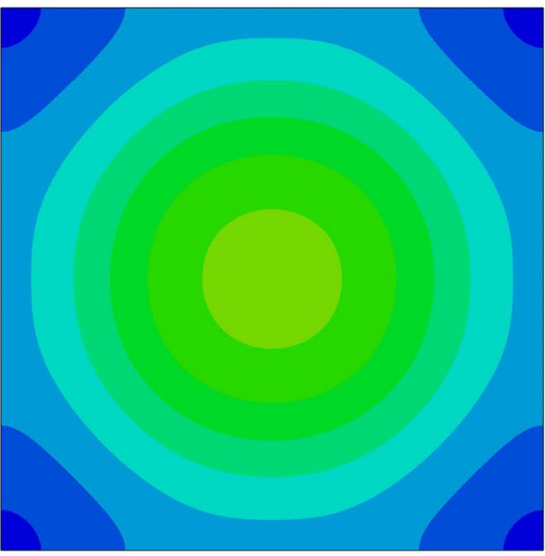

(a) G1

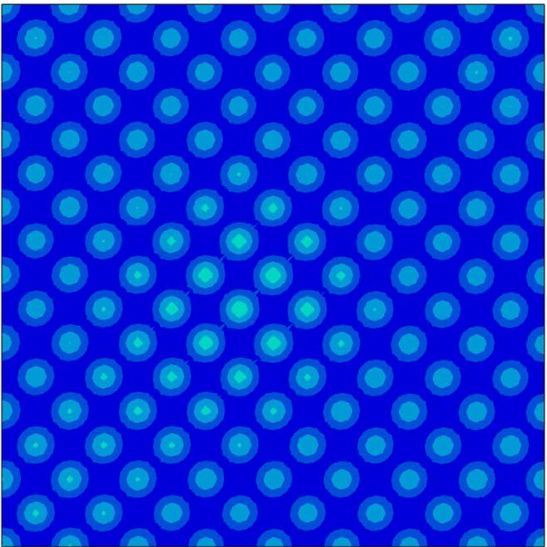

(d) $\mathrm{RHC}$

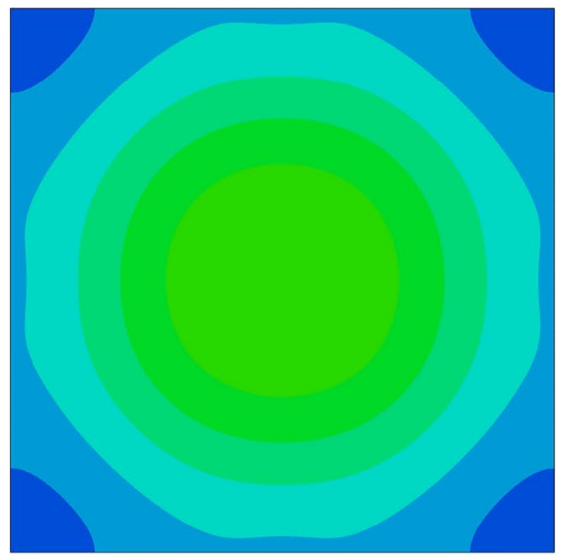

(b) G2

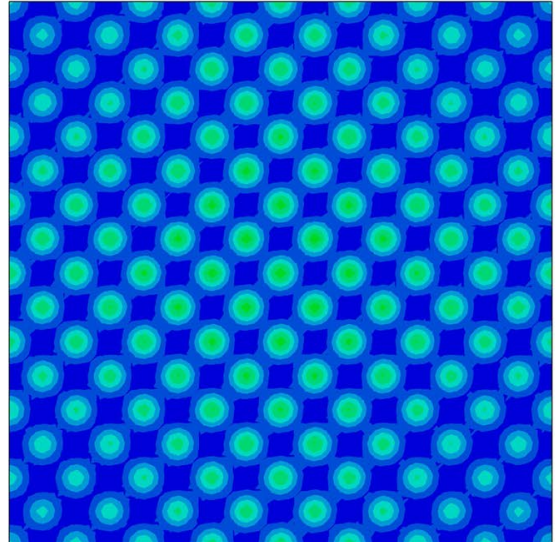

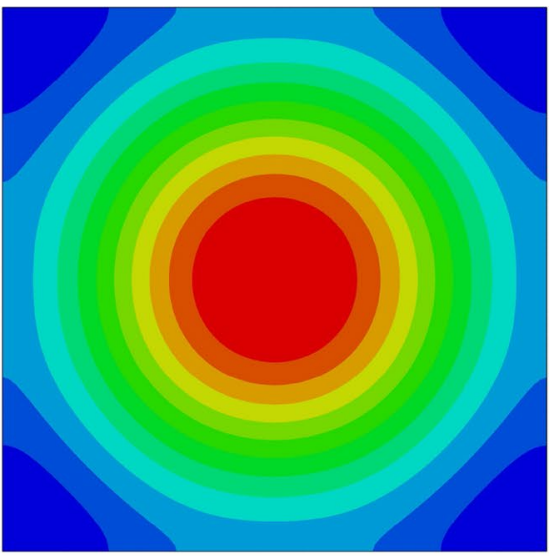

(c) G3

$\sigma[\mathrm{MPa}]$

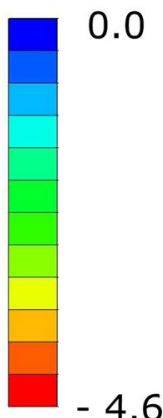

(e) $\mathrm{HC}$

Fig. 12 Maximum compression stress on the internal surface for different structures of protective devices

Funding Open access funding provided by Politecnico di Milano within the CRUI-CARE Agreement. Italian MIUR (PRIN project nr.2015LYYXA8 on Multiscale mechanical models for the design and optimization of micro-structured smart materials and metamaterials).

\section{Compliance with Ethical Standards}

Conflicts of interest The authors declare that they have no conflict of interest.

Open Access This article is licensed under a Creative Commons Attribution 4.0 International License, which permits use, sharing, adaptation, distribution and reproduction in any medium or format, as long as you give appropriate credit to the original author(s) and the source, provide a link to the Creative Commons licence, and indicate if changes were made. The images or other third party material in this article are included in the article's Creative Commons licence, unless indicated otherwise in a credit line to the material. If material is not included in the article's Creative Commons licence and your intended use is not permitted by statutory regulation or exceeds the permitted use, you will need to obtain permission directly from the copyright holder. To view a copy of this licence, visit http://creativecommons.org/licenses/by/4.0/.

\section{References}

1. Alderson KL, Pickles AP, Neale PJ, Evans KE (1994) Auxetic polyethylene: the effect of a negative Poisson's ratio on hardness. Acta Metall Mater 42(7):2261-2266

2. Ashby MF (2005) Materials selection in mechanical design, 3rd edn. Elsevier, Amsterdam

3. Bacigalupo A, Lepidi M, Gnecco G, Gambarotta L (2016) Optimal design of auxetic hexachiral metamaterials with local resonators. Smart Mater Struct 25(5):054009

4. Baldacchini $\mathrm{T}$ (2015) Three-dimensional microfabrication using two-photon polymerization. Elsevier, Amsterdam

5. Brito e Dias R, Coto NP, Batalha GF, Driemeier L (2018) Systematic Study of Ethylene-Vinyl Acetate (EVA) in the Manufacturing of Protector Devices for the Orofacial System. In: Biomaterials in regenerative medicine, chapter 14

6. Chulho Yang HDV, Chang Y (2018) Behavior of auxetic structures under compression and impact forces. Smart Mater Struct 27:02512

7. Coto NP, Meira JBC, Brito e Dias R, Driemeier L, de Oliveira Roveri G, Noritomi PY (2012) Assessment of nose protector for sport activities: finite element analysis. Dent Traumatol 28(2):108-113 
8. D'Alessandro L, Zega V, Ardito R, Corigliano A (2018) 3D auxetic single material periodic structure with ultra-wide tunable bandgap. Sci Rep 8(1):1-9

9. Dirrenberger J, Forest S, Jeulin D (2013) Effective elastic properties of auxetic microstructures: anisotropy and structural applications. Int J Mech Mater Des 9:21-33

10. Evans KE, Nkansah MA, Hutchinson IJ (1994) Auxetic foams: modelling negative Poisson's ratios. Acta Metallurgica Materialia 42:1289-1294

11. Gatt R, Vella Wood M, Gatt A, Zarb F, Formosa C, Azzopardi KM, Casha A, Agius TP, Schembri-Wismayer P, Attard L, Chockalingam N, Grima JN (2015) Negative Poisson's ratios in tendons: an unexpected mechanical response. Acta Biomaterialia 24:201-208

12. Guo Y, Zhang J, Chen L, Du B, Liu H, Chen L, Li W, Liu Y (2020) Deformation behaviors and energy absorption of auxetic lattice cylindrical structures under axial crushing load. Aerosp Sci Technol 98:105662

13. Huang J, Zhang Q, Scarpa F, Liua Y, Leng J (2017) In-plane elasticity of a novel auxetic honeycomb design. Compos Part B 110:72-82

14. Kolken HMA, Zadpoor AA (2017) Auxetic mechanical metamaterials. RSC Adv 7(9):5111-5129

15. Lakes R, Elms K (1993) Indentability of conventional and negative Poisson's ratio foams. J Compos Mater 27(12):1193-1202

16. Lu Z, Wang Q, Li X, Yang Z (2017) Elastic properties of two novel auxetic 3D cellular structures. Int J Solids Struct 124:46-56

17. Madke RR, Chowdhury R (2020) Anti-impact behavior of auxetic sandwich structure with braided face sheets and 3D re-entrant cores. Compos Struct 236(October 2019):111838
18. Malaviya P, Choudhary S (2018) Zygomaticomaxillary buttress and its dilemma. J Korean Assoc Oral Maxillofac Surg 44:151-158

19. Ren X, Das R, Tran P, Ngo TD, Xie YM (2018) Auxetic metamaterials and structures: a review. Smart Mater Struct 27(2):023001

20. Robert F (1985) Almgren: an isotropic three-dimensional structure with Poisson's ratio $=-1$. J Elast 15(4):427-430

21. Schwerdtfeger J, Heinl P, Singer RF, Körner C (2010) Auxetic cellular structures through selective electron-beam melting. Physica Status Solidi (B) Basic Res 247(2):269-272

22. Shepherd T, Winwood K, Venkatraman P, Alderson A, Allen T (2020) Validation of a finite element modeling process for auxetic structures under impact. physica status 1900197:1-14

23. Wang XT, Li XW, Ma L (2016) Interlocking assembled 3D auXetic cellular structures. Mater Des 99:467-476

24. Williams JL, Lewis JL (1982) Properties and an anisotropic model of cancellous bone from the proximal tibial epiphysis. J Biomech Eng 104(1):50

25. Yang L, Harrysson O, West H, Cormier D (2015) Mechanical properties of 3D re-entrant honeycomb auxetic structures realized via additive manufacturing. Int J Solids Struct 69-70:475-490

26. Yeganeh-Haeri A, Weidner DJ, Parise JB (1992) Elasticity of agr-cristobalite: a silicon dioxide with a negative Poisson's ratio. Science 257(5070):650-652

27. Zhang X, Yang D (2016) Mechanical properties of auxetic cellular material consisting of re-entrant hexagonal honeycombs. Materials 9(11):900 